\title{
Fermi Large Area Telescope detection of gamma-ray emission from the direction of supernova iPTF14hls
}

\author{
Qiang Yuan ${ }^{1,2}$, Neng-Hui Liao ${ }^{1}$, Yu-Liang Xin ${ }^{1,3}$, Ye Li $^{4}$, Yi-Zhong Fan ${ }^{1,2}$, Bing Zhang ${ }^{5}$, \\ Hong-Bo $\mathrm{Hu}^{6,3}$, Xiao-Jun $\mathrm{Bi}^{6,3}$ \\ ${ }^{1}$ Key laboratory of Dark Matter and Space Astronomy, Purple Mountain Observatory, Chinese \\ Academy of Sciences, Nanjing 210008,China; yuanq@pmo.ac.cn,yzfan@pmo.ac.cn \\ ${ }^{2}$ School of Astronomy and Space Science, University of Science and Technology of China, Hefei \\ 230026, Anhui, China \\ ${ }^{3}$ University of Chinese Academy of Sciences, 19A Yuquan Road, Beijing 100049, China \\ ${ }^{4}$ Kavli Institute of Astronomy and Astrophysics, Peking University, Beijing 100871, China \\ ${ }^{5}$ Department of Physics and Astronomy, University of Nevada Las Vegas, Las Vegas, NV 89154, \\ USA;zhang@physics.unlv.edu \\ ${ }^{6}$ Key Laboratory of Particle Astrophysics, Institute of High Energy Physics, Chinese Academy of \\ Sciences, Beijing 100049, China; huhb@ihep.ac.cn
}

\begin{abstract}
The remnant of supernova explosion is widely believed to be the acceleration site of high-energy cosmic ray particles. The acceleration timescale is, however, typically very long. Here we report the detection of a variable $\gamma$-ray source with the Fermi Large Area Telescope, which is positionally and temporally consistent with a peculiar supernova, iPTF14hls. A quasi-stellar object SDSS J092054.04+504251.5, which is probably a blazar candidate according to the infrared data, is found in the error circle of the $\gamma$-ray source. More data about the $\gamma$-ray source and SDSS J092054.04+504251.5 are needed to confirm their association. On the other hand, if the association between the $\gamma$-ray source and the supernova is confirmed, this would be the first time to detect high-energy $\gamma$-ray emission from a supernova, suggesting very fast particle acceleration by supernova explosions.

Subject headings: gamma-rays: observation — radiation mechanisms: non-thermal - cosmic rays
\end{abstract}

\section{Introduction}

Observations of $\gamma$-ray emission from supernova remnants (SNRs) prove that they are highenergy cosmic ray accelerators (Aharonian et al. 2004; Ackermann et al. 2013). Particle acceleration typically occurs in a very long timescale, e.g., hundreds to thousands of years, after the 
supernova explosion. This picture was consistent with the non-detection of $\gamma$-ray emission from the most nearby supernova, 1987A (Sood et al. 1988; Yoshii et al. 1996; Enomoto et al. 2003). It has been expected that fast particle acceleration might occur soon after supernova explosion if there were interactions between the ejecta and pre-existing dense material (Murase et al. 2011) or there was a young, powerful pulsar wind nebula (Murase et al. 2015). However, previous searches for $\gamma$-ray emission from supernovae located in dense circumstellar medium (Ackermann et al. 2015a) or from super-luminous supernovae (Renault-Tinacci et al. 2017) with the Fermi Large Area Telescope (Fermi-LAT; Atwood et al. 2009) have not led to a positive detection.

A very peculiar supernova, iPTF14hls, was discovered by the Intermediate Palomar Transient Factory on 2014 September 22.53 UT (Arcavi et al. 2017). This event has the identical spectra compared with a typical hydrogen-rich core-collapse supernova, but shows a very different light curve. The optical emission from iPTF14hls remains bright up to 600 days after the first detection, and experiences at least five rebrightenings in two years. What is even more surprising is that there was possibly an eruption $\sim 60$ years ago in 1954 at the same position of iPTF14hls. This event challenges the traditional understanding of the explosions of massive stars at the end of their lives (Woosley 2017).

Multi-wavelength observations are particularly important for understanding the nature of such a peculiar event. The observation in the X-ray band by Swift/XRT on 2015 May 23.05, which was about 244 days after the discovery, showed no detection of the source and gave an upper limit of luminosity of $L_{X}<2.5 \times 10^{41} \mathrm{erg} \mathrm{s}^{-1}$ in the $0.3-10.0 \mathrm{keV}$ band assuming a photon index of $\Gamma=2$ and a neutral hydrogen column density of $1.4 \times 10^{20} \mathrm{~cm}^{-2}$ (Arcavi et al. 2017). In 2015 May and 2016 June, the source was observed by the Arcminute Microkelvin Imager Large Array and the Very Large Array in the radio bands, resulting in no detection in all those observations Arcavi et al. 2017). These observations show evidence against interaction between the supernova ejecta and pre-existing material. However, a late time observation after $\sim 3$ years of the explosion revealed strong interaction between the shock and the circumstellar material Andrews \& Smith 2017).

Here we report the search for potential emission from iPTF14hls in the $\gamma$-ray band, using the data of the Fermi-LAT. We find a variable $\gamma$-ray source which is potentially coincident with iPTF14hls. This could be the first time to detect high-energy $\gamma$-ray emission from a supernova, if the association is true. However, the interpretation of the $\gamma$-ray emission seems to be difficult under the traditional scenario of supernova explosion, given the constraints from the X-ray and radio observations. On the other hand, we find a quasar within the error circle of the Fermi-LAT source, which is potentially a blazar candidate according to the infrared color diagram. No radio counterpart is found for this quasar, making its association with the $\gamma$-ray source still uncertain. More observations are necessary to firmly address the association of the $\gamma$-ray source with either the 
supernova or the blazar candidate (e.g., follow-up multi-wavelength monitorings of the candidate blazar and continuous Fermi-LAT observations of the $\gamma$-ray source).

\section{Fermi-LAT observations}

We use Fermi-LAT data recorded from 2008 August 4 to 2017 November 16, restricted to the Pass 8 Source class (evclass $=128 \&$ evtype $=3$ ). We select the data in a $14^{\circ} \times 14^{\circ}$ box region centered at the target source iPTF14hls with energies between $200 \mathrm{MeV} 1$ and $500 \mathrm{GeV}$. The events with zenith angles $>90^{\circ}$ are excluded to reduce the contamination from the Earth Limb. We use the Fermi-LAT Science Tools v10r0p5 2 and the standard binned likelihood analysis method gtlike to analyze the data. The sources in the third Fermi-LAT catalog (3FGL; Acero et al. 2015), together with the diffuse Galactic and isotropic background 3 gll_iem_v06.fits and iso_P8R2_SOURCE_V6_V06.txt, are included in the model. An additional field source at position $($ R.A., Dec. $)=\left(143.12^{\circ}, 53.08^{\circ}\right)$ has also been added, according to the residual map of the fitting.

We divide the data into two parts, before and after the explosion date of iPTF14hls, and perform the likelihood analysis, respectively. For the $\sim 6$ years of data before 2014 September 22, we find no significant emission at the position of iPTF14hls. The Test Statistic (TS) map for a $2^{\circ} \times 2^{\circ}$ region centered at iPTF14hls is shown in the left panel of Fig. 1. For the data after the supernova explosion, a clear $\gamma$-ray source appears in the data, as can be seen in the middle panel of Fig. 1 for the TS map assuming the same model as that used in the left one. Assuming a power-law point source right at the location of iPTF14hls, we find a TS value of $\sim 53$, and a spectral index of $2.03 \pm 0.16$. The best-fit position of the source using gtfindsrc turns out to be $($ R.A., Dec. $)=\left(140.21^{\circ}, 50.65^{\circ}\right)$, with a $68 \%(95 \%)$ error circle of $0.045^{\circ}\left(0.073^{\circ}\right)$. The distance between iPTF14hls and the best-fit position of the $\gamma$-ray source is about $0.065^{\circ}$. Therefore iPTF14hls is positionally consistent with the Fermi-LAT variable source at the $95 \%$ confidence level. We also perform an analysis of the data from 2015 August 4 to 2017 November 16, i.e., about one year after the explosion date (see below for the light curve analysis), which results in a best-fit position of $($ R.A., Dec. $)=\left(140.21^{\circ}, 50.67^{\circ}\right)$, and a $68 \%$ error circle radius of $0.051^{\circ}$. The separation between iPTF14hls and the best-fit position is $0.048^{\circ}$, within the $68 \%$ error circle (see

\footnotetext{
${ }^{1}$ The $200 \mathrm{MeV}$ lower threshold enables us to have a better angular resolution of selected events. Furthermore, as can be seen below, the $\gamma$-ray spectrum of this source is relatively hard, and there is not much emission at low energies. We have tested that choosing a $100 \mathrm{MeV}$ threshold gives similar results.

${ }^{2}$ http://fermi.gsfc.nasa.gov/ssc/data/analysis/software/

${ }^{3}$ http://fermi.gsfc.nasa.gov/ssc/data/access/lat/BackgroundModels.html
} 
the right panel of Fig. 1). In the following analysis, we will present the results based on the past three years of data (from 2014 September 22 to 2017 November 16), unless stated explicitly.

The $\gamma$-ray flux of the source is about $1.5 \times 10^{-9} \mathrm{~cm}^{-2} \mathrm{~s}^{-1}$ between 0.2 and $500 \mathrm{GeV}$. For a distance of $156 \mathrm{Mpc}$ (Arcavi et al. 2017), it corresponds to a $\gamma$-ray luminosity of $1.0 \times 10^{43} \mathrm{erg} \mathrm{s}^{-1}$. This value is comparable with the peak bolometric luminosity of iPTF14hls (Arcavi et al. 2017). Assuming a 3 year emission time, the total energy released in $\gamma$-rays is estimated to be about $10^{51}$ erg.
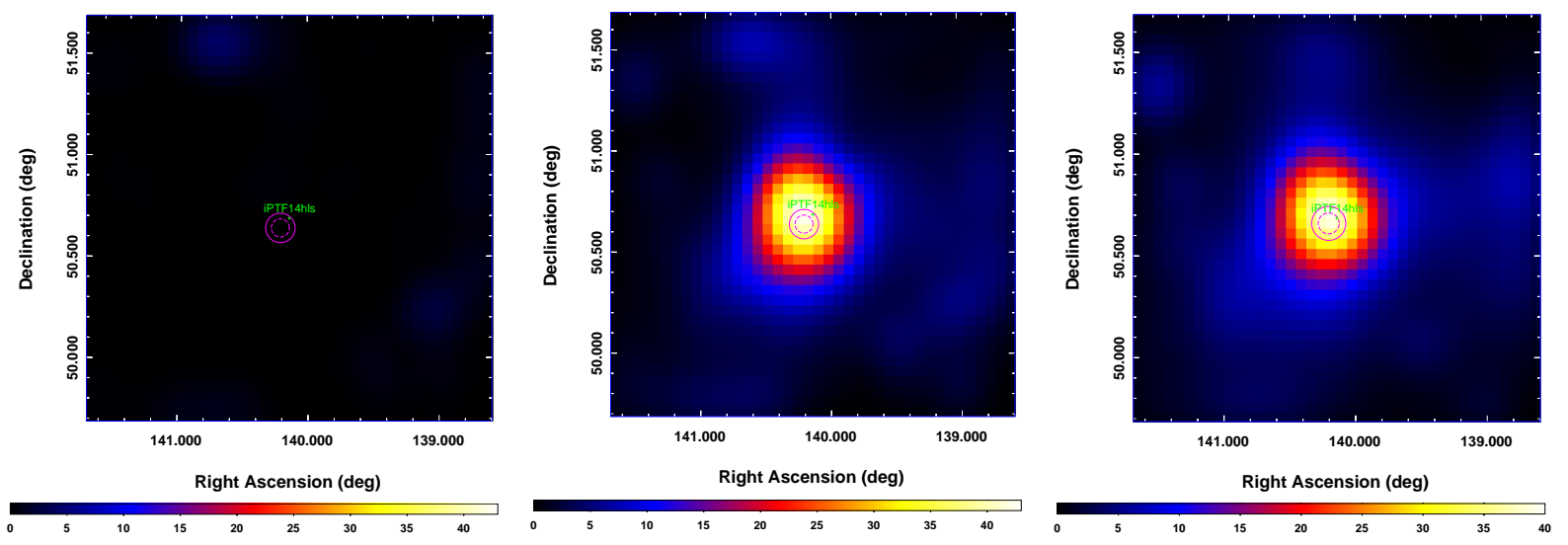

Fig. 1. - TS maps for a $2^{\circ} \times 2^{\circ}$ region centered at iPTF14hls, for the Fermi-LAT data before (left) and after (middle) 2014 September 22. The right panel shows the TS map for the past two years of data. All the maps are smoothed with a Gaussian kernel with a width of $0.2^{\circ}$. Magenta circles in these plots show the $68 \%$ (inner) and $95 \%$ (outer) error regions of the $\gamma$-ray localization.

To further address the variabilities of the $\gamma$-ray emission of the source, we derive the light curves of the $\gamma$-ray fluxes between 0.2 and $500 \mathrm{GeV}$, for 1-year and 2-month bins, which are shown in Fig. 2, For the time bins in which the TS values are smaller than 4, the $95 \%$ flux upper limits are given. We find that the source starts to emit $\gamma$-rays about 300 days after the explosion time of iPTF14hls, and the emission lasts for about 850 days. Weak emission may last for even longer time, but the significance becomes too low. We estimate the TS value of variabilities via $\mathrm{TS}_{\text {var }}=-2 \sum_{i} \ln \left[\mathcal{L}_{i}\left(F_{\text {const }}\right) / \mathcal{L}_{i}\left(F_{i}\right)\right]$, where $\mathcal{L}_{i}\left(F_{\text {const }}\right)$ and $\mathcal{L}_{i}\left(F_{i}\right)$ are the likelihoods in the $i$ th time bin for constant and variable flux assumptions (Nolan et al. 2012). The TS value of the variability during the period of 300 and 850 days after the supernova explosion is about 11.4 for the 2month binning light curve, which roughly corresponds to a $1.3 \sigma$ significance for 8 more degrees of freedom of the variable hypothesis. The emission is probably variable at even shorter timescales (see Fig. 3 for the light curve for 2-week bins). However, for most bins the TS values are too small to draw a clear conclusion. 

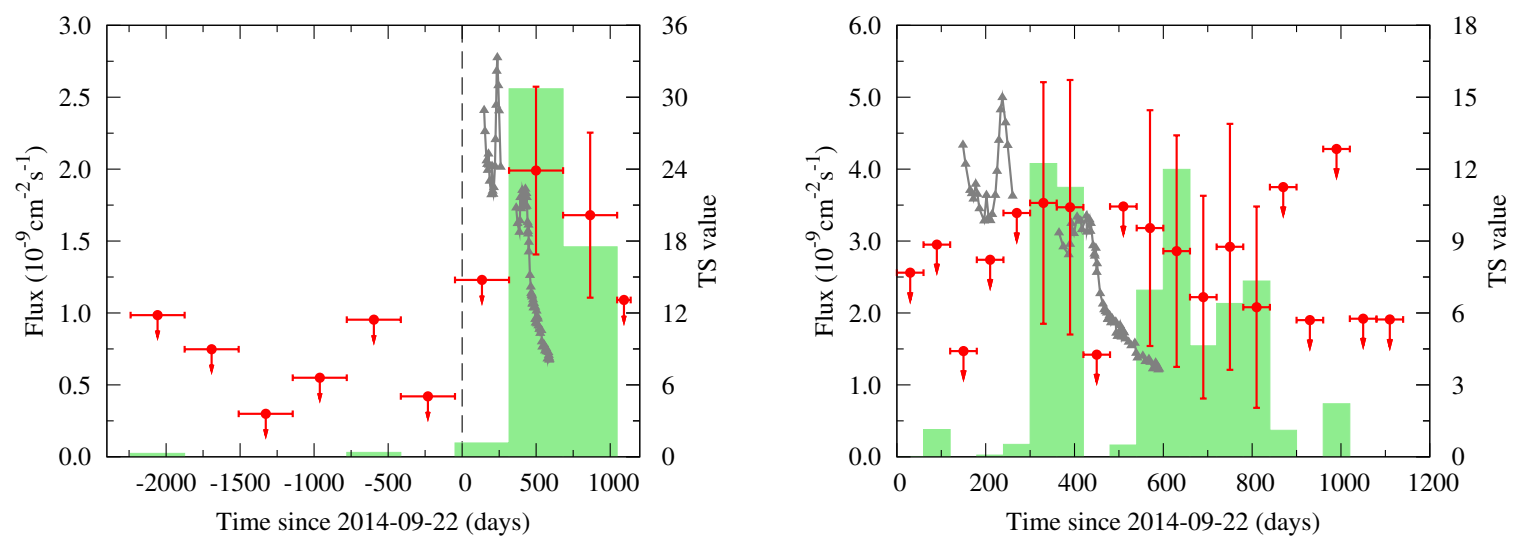

Fig. 2.- Light curves of $\gamma$-ray emission from the direction of iPTF14hls between 0.2 and 500 $\mathrm{GeV}$ in 1-year (left) and 2-month (right) bins. The zero point is adopted to be September 22, 2014. Shaded regions show the TS values (right axis). For TS values less than 4, the 95\% flux upper limits are presented. The bolometric luminosity with an arbitrary normalization of iPTF14hls deduced from the blackbody fits (Arcavi et al. 2017) is shown by gray triangles for comparison.

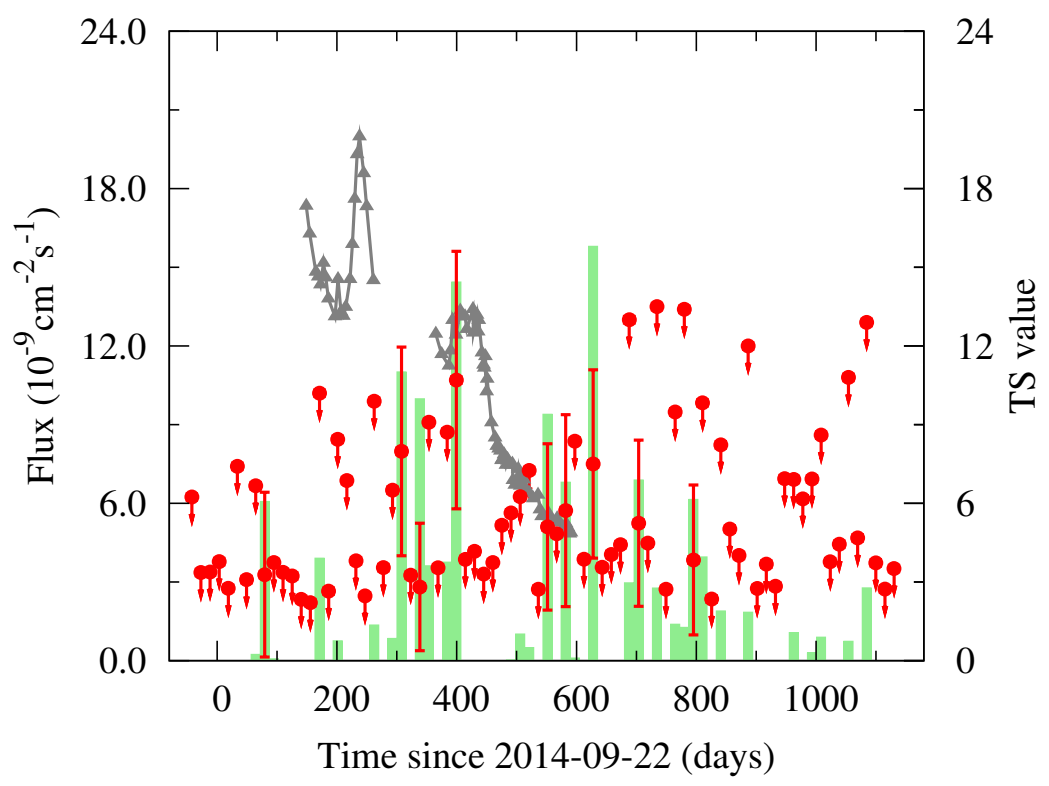

Fig. 3.- Same as Fig. 2 but for 2-week bins of the light curve.

The spectral energy distribution (SED) of the $\gamma$-ray emission for the data after the supernova explosion is presented in Fig. 4. The SED gives a flat spectrum which is consistent with that obtained in the global fit. 


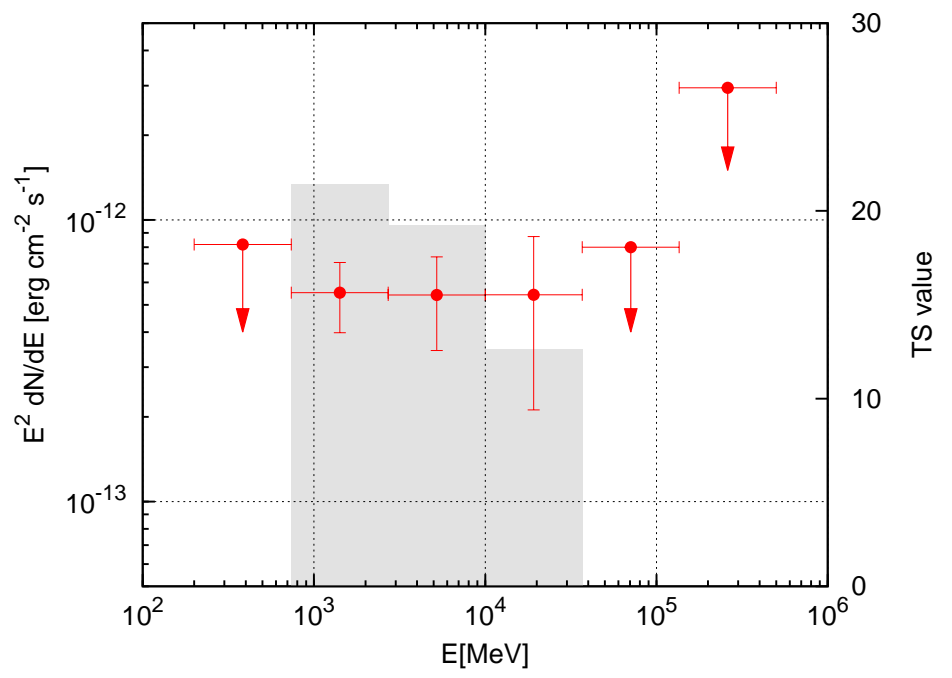

Fig. 4.- The SED of the $\gamma$-ray source, for data between 2014 September 22 and 2017 November 16. The gray shaded regions show the TS values (right axis).

\section{Discussion}

\subsection{SDSS J092054.04+504251.5}

A quasi-stellar object, SDSS J092054.04+504251.5, is found to be close to the $\gamma$-ray source, with an angular separation of $0.045^{\circ}$ from the best-fit position. The optical spectroscopy of SDSS J092054.04+504251.5 shows strong broad emission lines, suggesting that it is a quasar. This source is found to lie in the the so-called "WISE blazar stripe" in the color - color diagram of the WISE infrared data, and is thus a candidate blazar which is likely to be a $\gamma$-ray emitter (Massaro et al. 2012). If the $\gamma$-ray source found in this work is coincident with this quasar, its luminosity is estimated to be $\sim 9 \times 10^{46} \mathrm{erg} \mathrm{s}^{-1}$ adopting a redshift of $z=1.904$. The $\gamma$-ray luminosity and spectral index are consistent with, although lie in the edge of, that of Fermi-LAT flat-spectrum radio quasars (Ackermann et al. 2015b). However, blazars are typically radio loud. We have searched for possible radio emission from FIRST and NVSS, and do not find any counterpart of SDSS J092054.04+504251.5. This may be due to that this quasar is too distant and the radio surveys are not deep enough to reveal it. We also search for a possible radio counterpart of the $\gamma$-ray source, and do not find any source within its $95 \%$ error circle. The optical monitorings of SDSS J092054.04+504251.5 by the Intermediate Palomar Transient Factory gives an average $R$-band magnitude of 20.44 and a standard deviation of 0.15 between March 2009 and Januray 2015, which shows no significant variability. The data after 2015 are unavailable yet, which makes the comparison between optical and $\gamma$-ray variabilities impossible. In any case, we need more observations of SDSS J092054.04+504251.5 and/or the $\gamma$-ray source to establish their possible 
connection. Currently it is unclear whether they are associated with each other, and the association of the new $\gamma$-ray variable source with iPTF14hls is possible.

\subsection{Chance coincidence with a background $\gamma$-ray source}

We assume that the surface density distribution of Fermi-LAT sources is uniform, and the probability of observing a source in a particular position has a Poisson distribution. Then the probability to have an unrelated source "associated" with iPTF14hls is expected to be

$$
P_{\mathrm{ch}}=1-\exp \left[-\pi r_{\mathrm{eff}}^{2} \Sigma\left(>F_{\mathrm{th}}\right)\right]
$$

where $\Sigma\left(>F_{\text {th }}\right)$ is the surface density of Fermi-LAT sources with fluxes higher than $F_{\text {th }}, r_{\text {eff }}$ is an effective radius which takes into account the position uncertainties of the presumed Fermi-LAT counterpart $\left(\sigma_{\gamma}\right)$ and the target source iPTF14hls $\left(\sigma_{\text {opt }}\right)$, as well as the angular distance between them, $R_{0}$ (Bloom et al.2002). For our case, iPTF14hls is well located in the optical band, and thus $\sigma_{\mathrm{opt}}$ can be neglected. The effective radius is then $r_{\mathrm{eff}}=\left(R_{0}^{2}+4 \sigma_{\gamma}^{2}\right)^{1 / 2}$.

The cumulative numbers of the 3FGL sources as functions of fluxes are presented in Fig. 5 , In order to avoid the detection limits, we extrapolate the behaviors for fluxes between $2 \times 10^{-8}$ and $10^{-6}$ photon $\mathrm{cm}^{-2} \mathrm{~s}^{-1}$ to the average flux of the putative iPTF14hls counterpart converted to the energy range of $[0.1,100] \mathrm{GeV}, 3.0 \times 10^{-9}$ photon $\mathrm{cm}^{-2} \mathrm{~s}^{-1}$. The estimated number of $\gamma$ ray AGNs is 7164, corresponding to a number density of 0.17 degree $^{-2}$. For $R_{0}=0.065^{\circ}$ and $\sigma_{\gamma}=0.045^{\circ}$, the chance coincidence probability is estimated to be 0.007 . We also consider the $N(>$ Flux ) - Flux distributions for AGNs plus unidentified sources and all the 3FGL sources, and get chance coincidence probabilities 0.015 and 0.010 , respectively. Note that the temporal coincidence of the Fermi-LAT variable source with a background source may further decrease the chance coincidence probability by a factor of several (it is roughly the ratio of the whole FermiLAT observational time to the time period after the supernova explosion).

\subsection{Physical implications of the association with iPTF14hls}

Assuming that the Fermi-LAT source is associated with the supernova, we discuss possible physical implications of the $\gamma$-ray emission. The ejected mass by the supernova iPTF14hls was estimated to be several tens of solar masses, which corresponds to a total kinetic energy of

$$
E_{k} \sim \frac{M_{\mathrm{ej}} v_{\mathrm{sn}}^{2}}{2} \sim 1.8 \times 10^{52} \operatorname{erg}\left(\frac{M_{\mathrm{ej}}}{50 \mathrm{M}_{\odot}}\right)\left(\frac{v_{\mathrm{sn}}}{6000 \mathrm{~km} \mathrm{~s}^{-1}}\right)^{2},
$$




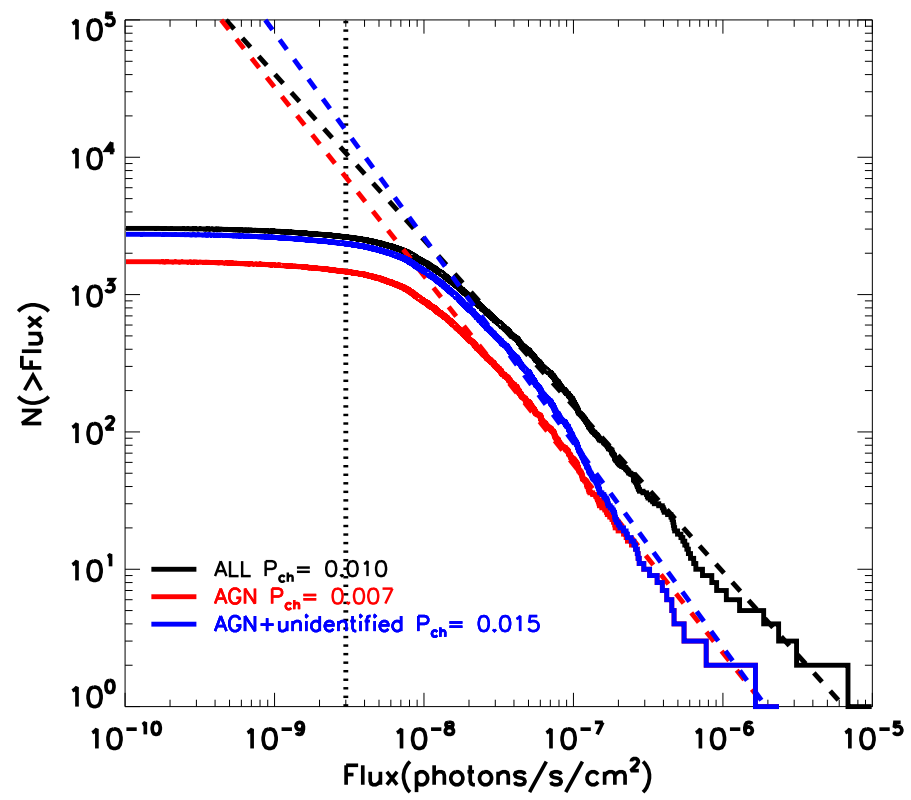

Fig. 5.- The cumulative numbers of sources versus the threshold fluxes for Fermi-LAT 3FGL sources (solid histograms), and the power-law fitting results of their high-flux trends (dashed lines). The black lines are for all the 3FGL sources, red are for AGNs, and blue are for AGNs with unidentified sources. The vertical dotted line shows the extrapolated $0.1-100 \mathrm{GeV}$ flux of the putative counterpart of iPTF14hls.

where $M_{\mathrm{ej}}$ is the ejected mass, and $v_{\mathrm{sn}}$ is the velocity of the ejecta (Arcavi et al. 2017). The corresponding gas density of the ejecta is estimated as $n_{\mathrm{gas}} \sim \frac{M_{\mathrm{ej}}}{4 \pi m_{p} R^{2} \Delta R}$, where $m_{p}$ is the proton mass, $R \sim v_{\mathrm{sn}} t \sim 1.8 \times 10^{16}(t / 1 \mathrm{yr}) \mathrm{cm}$ is the radius and $\Delta R$ is the width of the ejecta. Since $\Delta R<R$, we have $n_{\mathrm{gas}}>\frac{3 M_{\mathrm{ej}}}{4 \pi R^{3}} \approx 2.5 \times 10^{9}\left(M_{\mathrm{ej}} / 50 \mathrm{M}_{\odot}\right)(t / \mathrm{yr})^{-3} \mathrm{~cm}^{-3}$. The optical emission of iPTF14hls can be fitted with a diluted blackbody with a temperature of $5000-6000 \mathrm{~K}$, with a dilution factor $n_{\mathrm{ph}} / n_{\mathrm{bb}}$ varying from $\sim 1$ at $t \sim 100$ days and $\sim 10^{-4}$ at $t \sim 600$ days (inferred from Fig. 4 of Arcavi et al. 2017).

The GeV emission has a total energy of $\sim 10^{51} \mathrm{erg}$. We first assume that this emission is from the inverse Compton scattering (ICS) 4 off the optical photons by energetic electrons accelerated in a certain site. To boost the optical photons to $\mathrm{GeV}$ energies, the Lorentz factors of electrons

\footnotetext{
${ }^{4}$ The bremsstrahlung emission could be less dominated. The cooling rate due to ICS is about $10^{-16} \mathrm{GeV}^{\mathrm{cm}}{ }^{-3}$ $(E / \mathrm{GeV})^{2}\left(u / \mathrm{eV} \mathrm{cm}^{-3}\right)$ with $u$ being the energy density of photon field, and that due to bremsstrahlung radiation in neutral gas is about $10^{-15} \mathrm{GeV} \mathrm{cm}^{-3}(E / \mathrm{GeV})\left(n_{\text {gas }} / \mathrm{cm}^{-3}\right)$. For $t \sim 1 \mathrm{yr}$, the energy density of the photon field is about $3 \times 10^{10} \mathrm{eV} \mathrm{cm}^{-3}$, and the gas density is about $2.5 \times 10^{9} \mathrm{~cm}^{-3}$. For $E \gtrsim$ a few GeV, ICS cooling is dominant.
} 
are required to be $\gamma_{e} \sim 10^{4}$. The energy spectrum of electrons to produce a $E^{-2.0} \gamma$-ray spectrum is $d N / d E_{e} \propto E_{e}^{-3}$ in the slow cooling regime, whose total power is dominated by low energy particles. Therefore the energy fraction of high-energy electrons giving rise to the $\mathrm{GeV}$ emission should be very low (e.g., $10^{-4}$ for $E_{e}>10 \mathrm{GeV}$ compared with that for $E_{e} \gtrsim \mathrm{MeV}$ ). In case that there is a break of the energy spectrum of accelerated electrons below a few $\mathrm{GeV}$ like it is the case in the Milky Way (Strong et al. 2011), this fraction could be higher. Furthermore, we need a high enough acceleration efficiency to convert the kinetic energy to relativistic electrons. For typical supernovae observed in the Milky Way, the efficiency of electron acceleration is very low $\left(10^{-4} \sim 10^{-3}\right.$; Yang et al. 2014). The efficiency for the case of $\gamma$-ray bursts could be higher. However, we should keep in mind that there was no significant decrease of the expansion velocity of iPTF14hls (Arcavi et al. 2017), which limits the conversion fraction from the kinetic energy to the accelerated particles. Note that here we do not involve jets. If the $\gamma$-ray emission is collimated in a solid angle, the inferred total energy of $\gamma$-rays could be smaller and the required acceleration efficiency could be less extreme.

Another scenario to produce $\gamma$-rays is the decay of neutral pions produced by the hadronic $p p$ collision. To produce the $E^{-2} \gamma$-ray spectrum, the proton spectrum needs to be $d N / d E_{p} \propto E_{p}^{-2}$. The energy conversion efficiency of protons to $\gamma$-rays depends on the density of target material. The interaction timescale of protons in the hydrogen gas can be written as

$$
t_{\mathrm{pp}} \simeq \frac{1}{n_{\mathrm{gas}} \sigma_{\mathrm{pp}} c} \approx 3 \times 10^{7}\left(\frac{n_{\mathrm{gas}}}{\mathrm{cm}^{-3}}\right)^{-1} \mathrm{yr} .
$$

Here $\sigma_{\mathrm{pp}} \sim 40 \mathrm{mb}$ is the total interaction cross section of $p p$ collision for center-of-mass energy of tens of $\mathrm{GeV}$ (Patrignani \& et al. 2016). For a gas density higher than $2.5 \times 10^{9} \mathrm{~cm}^{-3}$ as estimated above, the protons can effectively convert their energy to $\gamma$-rays, with an efficiency of $\sim 33 \%$, the fraction of the neutral pion component. In such a case, the total energy of protons needs to be $\sim 10^{52}$ erg in order to give the observed GeV $\gamma$-ray energy (considering also that the energy band of the actual $\gamma$-ray emission may be wider than the Fermi-LAT coverage). In this case we may need a very high conversion efficiency of the supernova kinetic energy to the accelerated particle energy. Note that the energy conversion efficiencies obtained from observations of supernova remnants in the Milky Way are estimated to be about 10\% (Acero et al. 2010; Xin et al. 2017). The late time spectroscopic observations by Andrews \& Smith (2017) did find evidence of interactions between the supernova shock and the circumstellar medium, which supports the scenario of $\gamma$-ray production in $p p$ collisions. However, the lack of $\mathrm{X}$-ray and radio emission as well as significant deceleration of the ejecta at early time constrain the possible strong interactions Arcavi et al. 2017). Furthermore, even if there were interactions between the supernova ejecta and the pre-existing dense material, the offset between the $\gamma$-ray light curve and the optical one is still a challenge. We leave detailed modeling of this interaction scenario to future works.

In both cases discussed above, it could be very difficult for particles to get accelerated in such 
a high-density environment without thermalization. This could be a challenge of the modeling of the $\gamma$-ray emission from the supernova. In addition, for such a dense environment, the pair production optical depth of $\gamma$-ray photons with gas is

$$
\tau \sim \frac{\sigma_{\mathrm{pair}} M_{\mathrm{ej}}}{4 \pi R^{2} m_{p}} \sim 0.15\left(\frac{M_{\mathrm{ej}}}{50 \mathrm{M}_{\odot}}\right)\left(\frac{t}{\mathrm{yr}}\right)^{-2},
$$

where $\sigma_{\text {pair }} \sim 10 \mathrm{mb}$ is the pair production cross section for $\mathrm{GeV}$ photons (Wang et al. 2010). The $\mathrm{GeV}$ emission, if generated inside the ejected shell, may be subject to pair production absorbsion by the material, in particular at early time. This may explain why the $\gamma$-ray emission appears $\sim 300$ days after the explosion. Here we ignore the Thomson scattering, because the shell is expected to be optically thin and the radiation field is not sufficient to ionize the gas (Arcavi et al. 2017).

\section{Conclusion}

In this work we report the detection of a variable $\gamma$-ray source which is potentially coincident with the supernova iPTF14hls, using the Fermi-LAT data. The $\gamma$-ray source appears $\sim 300$ days after the explosion of iPTF14hls, and is still observable up to $\sim 850$ days. The search for $\gamma$-ray emission from 2008 August 4 to 2015 September 22 result in no detectable emission from this direction. The spectrum of the source is $\propto E^{-2}$ between 0.2 and $500 \mathrm{GeV}$, and the luminosity is $1.0 \times 10^{43}(d / 156 \mathrm{Mpc})^{2} \mathrm{erg} \mathrm{s}^{-1}$. The isotropic energy of the $\gamma$-ray emission is about $10^{51} \mathrm{erg}$.

There is a quasar, SDSS J092054.04+504251.5, in the error circle of the Fermi-LAT source, which is a blazar candidate according to the infrared color diagram of WISE. However, no radio counterpart is found from radio surveys FIRST and NVSS. The lack of multi-wavelength observations of SDSS J092054.04+504251.5 makes it difficult to conclusively address its connection with the $\gamma$-ray variable source. We also estimate the probability of chance coincidence of the $\gamma$-ray source with a background source based on the 3FGL catalog, and results in a low probability of $P_{\text {ch }} \lesssim 0.015$.

If the association between the $\gamma$-ray source and iPTF14hls is real, there are difficulties to model its $\gamma$-ray emission in the framework of particle acceleration in supernova ejecta produced shocks. The acceleration efficiency and the energy conversion efficiency of the accelerated particles to $\mathrm{GeV} \gamma$-ray emission need to be high. Furthermore, the acceleration of particles in the dense environment of the ejecta is also a big challenge. Anisotropic emission from e.g., a jet, may be necessary to explain the data (Soker \& Gilkis 2017).

We thank the anonymous referee for helpful suggestions on the paper. This work is supported by the National Natural Science Foundation of China under Grant Nos. 11525313, 11722328, and 
11703093, and the 100 Talents Program of Chinese Academy of Sciences.

\section{REFERENCES}

Acero, F., Aharonian, F., Akhperjanian, A. G., et al. 2010, A\&A, 516, A62

Acero, F., Ackermann, M., Ajello, M., et al. 2015, ApJS, 218, 23

Ackermann, M., Ajello, M., Allafort, A., et al. 2013, Science, 339, 807

Ackermann, M., Arcavi, I., Baldini, L., et al. 2015a, ApJ, 807, 169

Ackermann, M., Ajello, M., Atwood, W. B., et al. 2015b, ApJ, 810, 14

Aharonian, F. A., Akhperjanian, A. G., Aye, K., et al. 2004, Nature, 432, 75

Andrews, J. E., \& Smith, N. 2017, ArXiv e-prints, arXiv:1712.00514

Arcavi, I., Howell, D. A., Kasen, D., et al. 2017, Nature, 551, 210

Atwood, W. B., Abdo, A. A., Ackermann, M., et al. 2009, ApJ, 697, 1071

Bloom, J. S., Kulkarni, S. R., \& Djorgovski, S. G. 2002, AJ, 123, 1111

Enomoto, R., Ksenofontov, L. T., Katagiri, H., et al. 2003, ApJ, 591, L25

Massaro, F., D’Abrusco, R., Tosti, G., et al. 2012, ApJ, 752, 61

Murase, K., Kashiyama, K., Kiuchi, K., \& Bartos, I. 2015, ApJ, 805, 82

Murase, K., Thompson, T. A., Lacki, B. C., \& Beacom, J. F. 2011, Phys. Rev. D, 84, 043003

Nolan, P. L., Abdo, A. A., Ackermann, M., et al. 2012, ApJS, 199, 31

Patrignani, C., \& et al. 2016, Chinese Physics C, 40, 100001

Renault-Tinacci, N., Kotera, K., Neronov, A., \& Ando, S. 2017, ArXiv e-prints, arXiv:1708.08971

Soker, N., \& Gilkis, A. 2017, ArXiv e-prints, arXiv:1711.05180

Sood, R. K., Thomas, J. A., Waldron, L., et al. 1988, MNRAS, 234, 73P

Strong, A. W., Orlando, E., \& Jaffe, T. R. 2011, A\&A, 534, A54 
Wang, B., Yuan, Q., Fan, C., et al. 2010, Science China Physics, Mechanics, and Astronomy, 53, 842

Woosley, S. 2017, Nature, 551, 173

Xin, Y.-L., Guo, X.-L., Liao, N.-H., et al. 2017, ApJ, 843, 90

Yang, R.-Z., Zhang, X., Yuan, Q., \& Liu, S. 2014, A\&A, 567, A23

Yoshii, H., Baba, T., Kaneko, T., et al. 1996, ApJ, 472, 800 